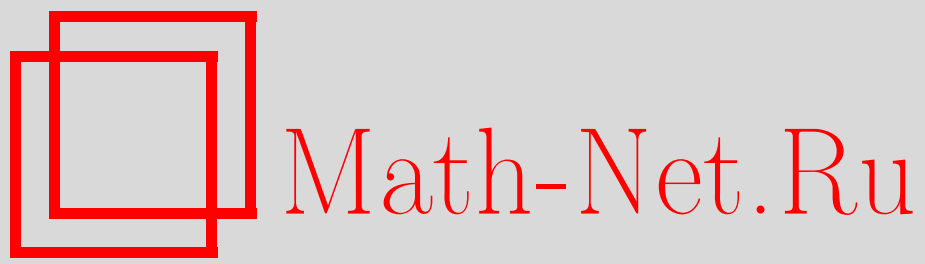

Н. Ю. Энатская, Глобализация анализа моделей размещения частиц по ячейкам, Вестн. Сам. гос. техн. ун-та. Сер. Физ.-мат. науки, 2021, номер 3, 571-587

DOI: https://doi.org/10.14498/vsgtu1857

Использование Общероссийского математического портала MathNet.Ru подразумевает, что вы прочитали и согласны с пользовательским соглашением

http://www . mathnet.ru/rus/agreement

Параметры загрузки:

IP : 54.147 .182 .235

26 апреля 2023 г., 13:56:36

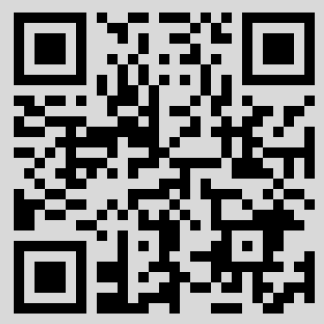


УДК 519.115

\title{
Глобализация анализа моделей размещения частиц по ячейкам
}

\author{
(C) Н. Ю. Энатская
}

Национальный исследовательский университет «Высшая школа экономики», Московский институт электроники и математики им. А. Н. Тихонова, Россия, 123458, Москва, ул. Таллинская, 34.

\begin{abstract}
Аннотация
Рассматривается общий подход к доасимптотическому анализу схем с разными качествами во всех сочетаниях по их различимости составляющих их элементов (ячеек и частиц). Для этого в каждой группе таких схем с общими ограничениями вместо непосредственного их изучения на основе учета специфики каждой схемы предлагается некоторый общий набор алгоритмических процедур пересчета результатов их доасимптотического анализа в схеме начиная со схемы с наибольшей дифференциацией их исходов последовательно для остальных схем группы с различиями в качестве одного элемента. Анализ каждой схемы проводится по традиционным и по ряду следующих новых направлений: построение случайного процесса формирования и нумерованного бесповторного перечисления исходов схемы в порядке их получения; нахождение числа исходов схемы; решение задачи нумерации для исходов схемы, состоящей в установлении взаимно однозначного соответствия между их видами и номерами; задание их вероятностного распределения и моделирования исходов схемы с этим вероятностным распределением.

В частности, отдельно изучаются случаи групп схем без ограничений размещения частиц и с ограничением (не более одной частицы в ячейке), приводящие к некоторым известным аналитическим результатам. При любых ограничениях в рассматриваемой группе схем их анализ проводится путем реализации алгоритмических процедур последовательного преобразования результатов анализа одной схемы группы для другой. Объединения в такие пары схем производятся по признаку различия качества одного их элемента.
\end{abstract}

Ключевые слова: размещение частиц по ячейкам, доасимптотический анализ.

Получение: 25 марта 2021 г. / Исправление: 18 августа 2021 г.

Принятие: 31 августа 2021 г. / Публикация онлайн: 30 сентября 2021 г.

\section{Научная статья}

(2) (7) Контент публикуется на условиях лицензии Creative Commons Attribution 4.0 International (https://creativecommons.org/licenses/by/4.0/deed.ru)

\section{Образец для цитирования}

Энатская Н. Ю. Глобализация анализа моделей размещения частиц по ячейкам // Вестн. Сам. гос. техн. ун-та. Сер. Физ.-мат. науки, 2021. Т. 25, № 3. С. 571-587. https://doi.org/10.14498/vsgtu1857.

\section{Сведения об авторе}

Наталия Юръевна Энатская (1) https://orcid.org/0000-0003-1241-7543

кандидат физико-математических наук, доцент; доцент; деп. прикладной математики; e-mail: nat1943@mail.ru 


\section{Введение}

Интерпретация многих комбинаторных задач в терминах размещений частиц по ячейками приводит их к простой наглядной постановке и находит многочисленные применения во многих научных областях, таких как теория вероятностей, математическая статистика, статистическая физика, теория автоматов и т.д., а для случаев схем размещений (без ограничений и с ограничением - не более одной частицы в ячейке) их числа исходов с разными качествами по различимости ячеек и частиц ${ }^{1}$ представляет основные комбинаторные схемы в книгах известных авторов [1-7].

В литературе по комбинаторике широко представлены асимптотические исследования комбинаторных схем и методы их проведения. Большое место в них отводится востребованным на практике схемам размещения частиц по ячейкам. Для нас и целей нашего исследования наибольший интерес представляет начальная схема размещений с наибольшей различимостью исходов при различии составляющих ее ячеек и частиц - схемы размещений с повторением без ограничений. В монографии [8] был проведен точный и асимптотический анализ статистик размещения; в частности, получены моменты и изучено асимптотическое поведение для числа пустых ячеек и моментов при разном асимптотическом поведении ее параметров.

Большинство доасимптотических исследований сводятся к элементарным результатам числа исходов основных комбинаторных схем. Исследования комбинаторных схем алгоритмического характера не привлекают особого внимания математиков из-за ограниченности области применения и громоздкости представлений и сводятся к решению отдельных комбинаторных задач. Но в связи с ростом производительности электронных вычислительных средств, усложнением комбинаторных схем и востребованностью информации о конкретных видах исходов актуальность таких исследований растет и требует выработки общих методов их проведения. Поэтому представляет интерес, например, монография [9], в которой проведено систематическое изложение задач перечислительной комбинаторики нахождения чисел исходов комбинаторных схем и конфигураций некоторых конечных совокупностей на общей основе использования производящих функций.

В настоящей работе вводится класс комбинаторных схем, для которых проводится доасимптотический анализ в возможных сочетаниях различимости ячеек и частиц новым авторским перечислительным методом, удовлетворяющим современным практическим требованиям построения итерационного случайного процесса бесповторного нумерованного перечисления всех исходов схемы (путем последовательного поединичного добавления на каждой итерации следующего элемента схемы или ранее изученного этапа перечисления предсостояний исходов схемы), т.е., по сути, формируются исходы схемы с управляемым вероятностным распределением. Графическое представление процесса перечисления исходов схемы, называемое методом графов, приводит к получению полной информации о них и дает наглядный способ вычисления вероятностей ее итоговых исходов по итерационным переходам в процессе (cм. [10]).

Часть результатов непосредственного анализа каждой схемы с учетом ее специфики со ссылками на конкретные работы приведены в [11].

\footnotetext{
13десь и далее различимость частиц или ячеек означает различие в их номерах.
} 
В перечислительном методе предложен прием универсального моделирования исхода комбинаторной схемы, состоящий в разыгрывании одним случайным числом его номера по принятому вероятностному распределению исходов схемы с дальнейшим получением его смоделированного вида по результату решения задачи нумерации в соответствии с его разыгранным номером (прямой задачи нумерации - нахождения вида исхода схемы по ее номеру при бесповторном нумерованном перечислении исходов схемы).

Рассматриваемый здесь общий подход, называемый глобализацией, применяется к исследованию схем размещения частиц по ячейкам в доасимптотической области изменения параметров через установление связей при разных качествах составляющих их элементов (ячеек и частиц). Он состоит в решении задач перечислительной комбинаторики, пересчете их результатов из схем с большей дифференциацией в исходах, проведении маркировок, упорядочении и отбраковке повторов. Смысл преобразований совокупностейв последовательном уменьшении дифференциации исходов схемы до различий в исследуемой схеме, где упорядочение (с указанием правила) частиц в ячейках или информации о заполнении ячеек производится при их неразличимостях; маркировка - преобразование в виде частот определенных введенных признаков их совпадений, например, составов ячеек по их уровням заполнения или исходов схемы, производится при неразличимости частиц или исходов; отбраковка повторов исходов схемы проводится для их бесповторного перечисления.

В анализе схемы для определения числа ее исходов будет использоваться операция суммы по перечислению исходов предшествующих процедур их итогового формировании по методу графов при его зависимости от их видов (с явным перечислением видов этих последовательных исходов) функций этой зависимости.

Для этого вводится индикаторная функция $I(Z)=\{0$ при $Z=0 ; 1$ при $Z>0\}$. Число исходов схемы определяется числом исходов итогового алгоритмического преобразования (отбраковки), приводящего к перечислению различимых исходов схемы. Это значит, что оно выражается суммой индикаторных функций от маркировок исходов предпоследнего преобразования по совпадению исходов для исследуемой схемы, что записывается через операцию сложения по перечислению всех ее различимых исходов слагаемых вида $I(Z)$ с аргументом $Z>0$.

Такой подход по сравнению с отдельным анализом каждой схемы имеет преимущество в естественности и общности исследований, но приводит к результатам алгоритмического характера.

Будут представлены как основные схемы размещения частиц по ячейкам (без ограничений), соответствующие всем вариантам варьирования сочетаний качеств элементов ячеек и частиц по их различимости, так и схемы с любыми, совпадающими в них ограничениями, которые будем изучать по направлениям, указанным в аннотации, со следующими описаниями схем:

- схема $A$ размещения различимых частиц по различимым ячейкам;

- схема $B$ размещения неразличимых частиц по различимым ячейкам;

- схема $C$ размещения различимых частиц по неразличимым ячейкам;

- схема $D$ размещения неразличимых частиц по неразличимым ячейкам, так и их аналоги с ограничениями. 
Отдельно будут анализироваться частные случаи основных схем при отсутствии ограничений в них и при размещении в каждую ячейку не более одной частицы, где наряду с указанными алгоритмическими процедурами получения исходной информации о бесповторном перечислении их исходов для части схем аналитически определяются числа их исходов.

Схемы будут рассматриваться в порядке их алфавитного обозначения, что обусловлено логикой последовательных пересчетов результатов анализа схем, связанной с поединичным уменьшением признаков различий в их исходах, кроме порядка изучения схем $B$ и $C$.

\section{1. Виды исходов схем, их перечисления и известные числа исходов}

1.1. Схемы без ограничений. 2 Виды и числа исходов схем $A$ и $B$ изучены, перечисления построены в $[11,12]$, а для схем $C$ и $D$ будут проанализированы позже, как частные случаи схемы $A$.

Вид исхода схемы $A$ (известной как схема размещения с повторением) представляется составами номеров частиц в ячейках в порядке ячеек $\bar{m}=$ $=\left(\bar{m}_{1}, \ldots, \bar{m}_{n}\right)$, где $\bar{m}_{i}-$ состав номеров частиц в $i$-той ячейке, $i=\overline{1, n}$. Число ее исходов $N_{A}=n^{r}$ известно. Перечислительный анализ схемы $A$ проведен в [12] (без ограничений и с ограничением - без пустых ячеек) по следующим направлениям: прямое перечисление ее исходов; решение задачи нумерации; введение вероятностного распределения ее исходов и построение процедуры моделирования.

Вид исхода схемы $B$ (известной как схема сочетания с повторением) представляется последовательностью уровней заполнения ячеек в порядке ячеек $\bar{r}=\left(r_{1}, \ldots, r_{n}\right)$. Число ее исходов $N_{B}=C_{n+r-1}^{r}$ известно. Перечислительный анализ схемы $B$ следует из анализа схемы сочетаний с его результатами в [11] (в силу взаимно однозначного соответствия их исходов с исходами схемы сочетаний с повторением) по следующим направлениям: прямое перечисление ее исходов; решение задачи нумерации; введение вероятностного распределения ее исходов и построение процедуры моделирования.

Перечисление исходов схемы $B$ получается из перечисления исходов схемы $A$ проведением маркировки ее исходов по совпадению уровней заполнения ячеек в порядке ячеек с последующей отбраковкой повторов с результатом бесповторного перечисления наборов уровней заполнения ячеек в порядке ячеек $\left\{\bar{r}^{*}\right\}=\left(r_{1}^{*}, \ldots, r_{n}^{*}\right)$.

1.2. Схемы с ограничением (ячейка вмещает одну частицу при $\boldsymbol{r} \leqslant \boldsymbol{n})$. Вид исхода схемы $A$ (известной как схема размещения) представляется номерами частиц в ячейках в порядке ячеек $\bar{m}=\left(m_{1}, \ldots, m_{r}\right)$. Число ее исходов $N_{A}=A_{n}^{r}$ известно. Результаты перечислительного анализа схемы $A$ приведены в [11] по следующим направлениям: прямое перечисление ее исходов; решение задачи нумерации; введение вероятностного распределения ее исходов и моделирование по результату решения прямой задачи нумерации в схеме размещений.

Вид исхода схемы $B$ (известной как схема сочетаний) представляется номерами непустых ячеек в порядке роста их номеров. Число ее исходов

\footnotetext{
2 Здесь и далее $n$ - число ячеек, $r$ - число частиц.
} 
$N_{B}=C_{n}^{r}$ известно. Результаты перечислительного анализа схемы $B$ приведены в [11] по следующим направлениям: прямое перечисление ее исходов; решение задачи нумерации; введение вероятностного распределения ее исходов и моделирование по результату решения прямой задачи нумерации в схеме сочетаний.

Исход схемы $C$ один $\left(N_{C}=1\right)$ и имеет вид набора номеров попавших в ячейки частиц $(0, \ldots, 0,1,2, \ldots, r)$ в заранее заданном порядке, например, в порядке роста номеров частиц, где нули означают пустые ячейки.

Исход схемы $D$ один $\left(N_{D}=1\right)$ и имеет вид маркировки по совпадению уровней заполнения ячеек $(n-r, r)$ исхода схемы $C$, где $(n-r)$ - число пустых ячеек, а $r$ - число ячеек, содержащих по одной частице.

Таким образом, вопрос о перечислениях, видах и числах исходов схем размещения частиц по ячейкам с ограничением данного вида при всех качествах ячеек и частиц полностью изучен.

\section{2. Вычисление чисел исходов схем размещения частиц по ячейкам}

По способу вычисления чисел исходов схем будем различать схемы без ограничений и с ограничениями.

2.1. Структура формирования исходов схемы $\boldsymbol{A}$ и числа исходов схем $\boldsymbol{B}, \boldsymbol{C}, \boldsymbol{D}$ без ограничений. В процедуре перечисления исходов схемы $A$, приведенной в [11], структура исходов формируется поитерационно с учетом различимости ячеек и частиц. При этом разделение этого учета для составляющих его элементов (ячеек и частиц) для пересчета из нее перечисления исходов схем $B, C, D$ не представляется возможным. Поэтому построим другую разделяемую структуру перечисления исходов схемы $A$ в виде последовательных действий по отдельному учету признаков их различимости.

Число $N_{A}=n^{r}$ схемы $A$ учитывает все разные составы различимых частиц в ячейках и все разные порядки этих составов частиц по ячейкам в схеме размещений с повторениями.

Под структурой исходов схемы $A$ понимаются последовательные процедуры по характеру учета условий их перечисления, что в дальнейшем даст возможность пересчета чисел исходов остальных схем из перечисления исходов схемы $A$ выбором соответствующих им условий.

Теорема 1. Пусть $\bar{r}=\left(r_{1}, \ldots, r_{n}\right)$-маркировка по совпадению ячеек размещенных частии, т.е. уровни заполнения ячеек в исходах схемь $A$, a $\bar{\mu}=\left(\mu_{1}, \ldots, \mu_{p}\right)$-маркировка $\bar{r}$ по совпадению уровней заполнения ячеек. Здесъ $\mu_{j}$ - положительная частота ячеек с уровнем заполнения ячеек $j$, $j=\overline{1, p} ; r_{1}, \ldots, r_{n}, \mu_{1}, \ldots, \mu_{p}-$ челые числа, для которых $r_{1}+\cdots+r_{n}=r$, $\sum_{j=1}^{p} \mu_{j}=n, \sum_{j=1}^{p} j \mu_{j}=r$. Тогда справедливо представление с операцией (суммирования) по перечислению (*) разных наборов уровней заполнения ячеек $\left\{\bar{r}^{*}\right\}=\left\{\left(r_{1}^{*}, \ldots, r_{n}^{*}\right)\right\}$, упорядоченных по возрастанию:

$$
N_{A}=n^{r}=\sum_{(*)} \frac{r !}{r_{1}^{* !} \cdots r_{n}^{* !}} \cdot \frac{n !}{\mu_{1} ! \cdots \mu_{p} !} .
$$

Доказательство. Число исходов схемы $A$ (схемы размещений с повторением) $N_{A}=n^{r}$ можно интерпретировать как число размещений $r$ раз- 
личимых частиц по $n$ различимым ячейкам по перечислению всех разных наборов уровней заполнения ячеек $\left\{\bar{r}^{*}\right\}$ всех вариантов размещения частиц по каждому набору их уровней заполнения в каждом их порядке (с совпадающими количествами в каждом порядке) и во всех различимых порядках.

Первый перебор всех наборов $\left\{\bar{r}^{*}\right\}$ в формуле (1) соответствует сумме по $(*)$, а следующие два по каждому набору из $(*)$ - схемам перестановок с повторением в количествах первого и второго сомножителей в (1): размещения частиц по ячейкам в одном порядке уровней их заполнения, учитывающем различимость частиц, и перебора всех различимых порядков уровней заполнения ячеек делением ячеек на группы размерами чисел совпадающих уровней заполнения, учитывающим различимость ячеек, что и приводит к формуле (1).

Явное перечисление элементов множества $(*)$ представляет собой все исходы схемы $D$ без ограничений с числом $N_{D}$ и, когда их визуальное перечисление затруднительно, определяется по шагам следующего алгоритма.

Алгоритм.

1) Перечисляем все исходы схемы $B$ без ограничений как исходы схемы сочетаний с повторением выбором $(n-1)$ мест $\bar{t}=\left(t_{1}, \ldots, t_{n-1}\right)$ среди $(n+r-1)$ для $r$ частиц и $(n-1)$ внутренних перестановок между ячейками числом способов $N_{B}=C_{n+r=1}^{n-1}$, что с пересчетом из них уровней заполнения ячеек при $i=\overline{2, n-1}$ дает $r_{i}=t_{i}-t_{i-1}-1 ; r_{1}=t_{1}-1$; $t_{n}=n+r-1-t_{n-1}$

2) упорядочиваем исходы шага 1) в терминах уровней заполнения ячеек в порядке их возрастания;

3) отбраковываем в исходах шага 2) повторы - получаем все исходы схемы $D$ без ограничений (число $N_{D}$ определяется визуально).

ПримеР 1. Пусть $n=2, r=3$. Тогда $N_{C}=C_{4}^{1}=4$ исхода номеров мест перегородки вида: (1), (2), (3), (4), что соответствует 4-м исходам схемы $B$ в терминах уровней заполнения ячеек: $(0,3),(1,2),(2,1),(3,0)$. После упорядочения и отбраковки повторов получаем два исхода схемы $D:(0,3),(1,2)$.

Вычисление $N_{A}$ с небольшими значениями параметров $n$ и $r$ схемы $A$ можно производить в следующем порядке:

1) визуально выпишем все $N_{A}=n^{r}$ исходов схемы;

2) по всем исходам 1) проведем их маркировку по уровням заполнения ячеек в порядке роста минимальных уровней заполнения в них и с отбраковкой повторов - получаем наборы $\left\{\bar{r}^{*}\right\}$;

3) по всем исходам 2) проведем их маркировку по совпадению наборов уровней заполнения ячеек в порядке роста этих уровней;

4) произведем вычисление числа исходов схемы $A$ по формуле $(1)$, сравнивая со значением $N_{A}=n^{r}$.

Приведем примеры вычисления по вышеприведенным шагам числа $N_{A}$ при небольших значениях параметров схемы, сверяя их с результатами $N_{A}=$ $=n^{r}$ и визуальным перечислением исходов схемы $A$.

ПримеР 2. Пусть $n=2, r=2$.

1) Визуально перечислим все $n^{r}=2^{2}=4$ исхода схемы $A:(0,(1,2)),((1,2), 0)$, $(1,2),(2,1)$;

2) $\{\bar{r}\}=(0,2),(2,0),(1,1),(1,1)$, откуда после упорядочения и отбраковки 
получаем $\left\{\bar{r}^{*}\right\}=\left\{\left(r_{1}^{*}, \ldots, r_{n}^{*}\right)\right\}=((0,2),(1,1)) ;$

3) из результатов 2) находим $\{\bar{\mu}\}=((1,1),(2))$;

4) по (1) определяем

$$
N_{A}=\frac{2 !}{0 ! \cdot 2 !} \cdot \frac{2 !}{1 ! \cdot 1 !}+\frac{2 !}{1 ! \cdot 1 !} \cdot \frac{2 !}{2 !}=4,
$$

что совпало с полученными выше результатами.

ПримеР 3. Пусть $n=3, r=2$.

1) Визуально перечислим все $n^{r}=3^{2}=9$ исходов схемы $A$ : $(0,0,(1,2))$, $(0,(1,2), 0),((1,2), 0,0),(0,1,2),(1,0,2),(1,2,0),(0,2,1),(2,0,1),(2,1,0)$;

$2)$ из $\{\bar{r}\}$ после упорядочения и отбраковки получаем $\left\{\bar{r}^{*}\right\}=((0,0,2)$, $(0,1,1))$;

3) из результатов 2) находим $\{\bar{\mu}\}=\left\{\left(\mu_{i_{1}}, \ldots, \mu_{i_{p}}\right)\right\}=(2,1),(1,2)$;

4) по (1) вычисляем

$$
N_{A}=\frac{2 !}{0 ! \cdot 0 ! \cdot 2 !} \cdot \frac{3 !}{2 ! \cdot 1 !}+\frac{2 !}{0 ! \cdot 1 ! \cdot 1 !} \cdot \frac{3 !}{1 ! \cdot 2 !}=9,
$$

что совпало с полученными выше результатами.

Теорема 2. В условиях и обозначениях теоремы 1 для схем $B, C, D$ численности их исходов $N_{B}, N_{C}, N_{D}$ определяются по формулам

$$
\begin{gathered}
N_{B}=\sum_{(*)} \frac{n !}{\mu_{i_{1}} ! \cdots \mu_{i_{p}} !} \\
N_{C}=\sum_{(*)} \frac{r !}{r_{1}^{* !} \cdots r_{n}^{*} !} \\
N_{D}=\sum_{(*)} 1 .
\end{gathered}
$$

Доказ ательство. Формулы (2)-(4) для чисел исходов схем $B, C, D$ следуют из интерпретаций сомножителей формулы (1) в доказательстве теоремы 1:

- для получения $N_{B}$ при неразличимости частиц в (1) первый сомножитель заменяется на 1 , что приводит к (2);

- для получения $N_{C}$ при неразличимости ячеек в $(1)$ второй сомножитель заменяется на 1, что приводит к (3);

- для получения $N_{D}$ при неразличимости частиц в (1) оба сомножителя заменяется на 1, что приводит к (4).

2.2. Перечисление исходов схем $B, C, D$ из исходов схемы $A$. Указанные перечисления можем теперь производить двумя способами:

1) получением исходов схемы из исходов схемы $A$ по соответствующим алгоритмическим процедурам;

2) непосредственным получением их итогового итерационного (через стрелочку между итерациями) перечисления методом графов [10].

Проверим полученное число исходов по формулам теоремы 2.

Пример 4. Пусть $n=2, r=2$. Выпишем исходы схемы $A$ по примеру 1: $(0,(1,2)),((1,2), 0),(1,2),(2,1) ; N_{A}=4$. 
Cxema $B$ :

1) $(0,2),(1,1),(2,0)$;

2) $(0,0) \rightarrow(0,1) \rightarrow(0,2),(1,1),(2,0)$;

$$
N_{B}=\frac{2 !}{1 ! \cdot 1 !}+\frac{2 !}{2 !}=3,
$$

что совпадает с полученным результатом их перечисления и по формуле числа исходов для сочетания с повторением $C_{n+r-1}^{r}=C_{3}^{2}=3$. Схема $C$ :

1) $(0,(1,2)),(1,2)$;

2) $(0,0) \rightarrow(0,1) \rightarrow(0,(1,2)),(1,2)$;

$$
N_{C}=\frac{2 !}{0 ! \cdot 2 !}+\frac{2 !}{2 !}=2,
$$

что совпадает с полученным результатом их перечисления. Схема $D$ :

1) $(0,2),(1,1)$

2) $(0,0) \rightarrow(0,1) \rightarrow(0,(1,2)),(1,2)$;

$$
N_{D}=1+1=2
$$

что совпало с полученным в 2) результатом.

2.3. Перечисления и числа исходов схем $A, B, C, D$ с любыми ограничениями. Далее будем использовать следующие обозначения: $\bar{m}=\left(\bar{m}_{1}, \ldots, \bar{m}_{n}\right)$ - составы частиц в ячейках в порядке ячеек от 1 до $n$ (вид исхода схемы $A) ;\{\bar{m}\}$ - все исходы схемы $A ; \bar{m}^{*}=\left(\bar{m}_{1}^{*}, \ldots, \bar{m}_{n}^{*}\right)$ - составы частиц в ячейках в порядке роста минимальных номеров частиц в них от 1 до $n$ (вид исхода схемы $C) ;\{\bar{M}\}$ - результат отбраковки в $\left\{\bar{m}^{*}\right\}$ повторов исходов (все разные исходы схемы $C) ; \bar{r}=\left(r_{1}, \ldots, r_{n}\right)$ - уровни заполнения ячеек в порядке ячеек от 1 до $n$ (результат маркировки $\bar{m}$ по совпадению ячеек размещения в них частиц; вид исхода схемы $B) ;\left\{\bar{r}^{*}\right\}=\left\{\left(r_{1}^{*}, \ldots, r_{n}^{*}\right)\right\}-$ результат отбраковки в $\{\bar{r}\}$ повторов исходов (все разные исходы схемы $B$ ); $\bar{\mu}$ - частоты уровней заполнения ячеек от 0 до $r$ (результат маркировки $\bar{r}$ по совпадению их размеров повторов исходов - составов частиц в ячейках по совпадению их размеров; вид исхода схемы $D) ;\left\{\bar{\mu}^{*}\right\}$ - результат отбраковки в $\{\bar{\mu}\}$ повторов исходов - в количестве разных исходов схемы $D$.

В условиях ограничения число $N_{A}$ и перечисление исходов схемы $A$ будем считать известными.

2.3.1. Перечисление исходов схем $\boldsymbol{B}, \boldsymbol{C}, \boldsymbol{D}$. Ограничения в схемах приводят к удалению в соответствующих схемах без ограничений не удовлетворяющих им исходов. Тогда при совпадающих во всех схемах ограничениях будем находить перечисления исходов остальных схем пересчетом из схемы $A$ в соответствии с данными для них во введении алгоритмическими процедурами, определяя для каждой близкую схему, исходы которой за наименьшее число алгоритмических процедур могут преобразовываться в исходы изучаемой. В нашей группе схем - это схема $A$ для схем $B$ и $C$ и схемы $B$ или $C$ для схемы $D$. 
При различии в качестве частиц проводится маркировка составов заполнения ячеек в виде их уровней заполнения по совпадению ячеек размещения частиц с последующей отбраковкой повторов, а при различии в качестве ячеек - упорядочение информации о частицах в ячейках в заранее определенном их порядке с последующей отбраковкой повторов.

Получение исходов схемъ $B$ из исходов схемы $A$ :

1) маркируем исходы $\{\bar{m}\}$ по совпадению ячеек размещения частиц и получаем наборы уровней заполнения частиц $\{\bar{r}\}$ в порядках ячеек;

2) проводим отбраковку повторов в $\{\bar{r}\}$ и получаем $\left\{\bar{r}^{*}\right\}$ - бесповторное перечисление исходов схемы $B$.

Получение исходов схемы $C$ из исходов схемы $A$ :

1) упорядочиваем $\{\bar{m}\}$ по росту минимальных элементов в ячейках и получаем составы $\left\{\bar{m}^{*}\right\}$ частиц в ячейках;

2) проводим отбраковку повторов в результате 1) и получаем $\{\bar{M}\}-$ бесповторное перечисление исходов схемы $C$.

Получение исходов схемы $D$ из исходов схемы $B$ :

1) маркируем исходы $\left\{\bar{r}^{*}\right\}$ схемы $B$ по совпадению уровней заполнения ячеек в порядке роста уровней от 0 до $r$ и получаем $\{\bar{\mu}\}$;

2) проводим отбраковку повторов исходов вида $\{\bar{\mu}\}$ и получаем $\left\{\bar{\mu}^{*}\right\}-$ бесповторное перечисление исходов схемы $D$.

\subsection{2 Числа исходов схем $B, C, D$.}

Tеорема 3. В данных обозначениях для схем $B, C, D$ числа их исходов $N_{B}, N_{C}, N_{D}$ определяются формулами

$$
N_{B}=\sum_{(\{T\})} I(T),
$$

где $T$ - результат маркировки исходов $\{\bar{r}\}$ по их совпадению;

$$
N_{C}=\sum_{\left(\left\{M^{*}\right\}\right)} I\left(M^{*}\right)
$$

где $M^{*}$ - результат маркировки исходов $\{\bar{M}\}$ по их совпадению;

$$
N_{D}=\sum_{(\{V\})} I(V)
$$

где $\bar{V}$ - результат маркировки исходов $\left\{\bar{\mu}^{*}\right\}$ по их совпадению.

Доказ а тельство. Формулы (5)-(7) для чисел исходов схем $B, C, D$ следуют из завершающего шага отбраковки повторов в алгоритмических процедурах получения их перечисления из исходов близких схем с повторами. Тогда формульная запись числа разных исходов в их совокупности с повторами представляется суммой индикаторных функций от результатов маркировки по их совпадениям вместо отбраковки повторов при их перечислении. А так как маркировки на совпадения проводятся по бесповторным исходам, числа исходов схем совпадают с размерностями маркировок. 


\section{3. Задача нумерации для исходов схем}

Решение задачи нумерации в схеме $A$ (без ограничений) - схеме размещений с повторением - приведена в [12] в прямой и обратной постановках. Пересчет этих результатов решения задачи нумерации схемы $A$ для других схем размещения частиц по ячейкам будем производить в связи с двумя типами отличий в них от начальной схемы: введением ограничений или изменением качества ее элементов, приводящих к схемам $B, C, D$. Первое отличие приводит к рассмотрению части исходов начальной схемы, а второе - к группированию ее исходов по причине уменьшения их дифференциации.

Оказывается, что в обоих случаях задачи пересчета результатов решения задачи нумерации в начальной схеме $S$ к исследуемой $S^{*}$ можно рассматривать, считая ее исходы частью исходов схемы $S$, т.к. при пересчете результатов задачи нумерации в близкой схеме в качестве исходов схемы $S^{*}$ можно брать первые исходы групп в их порядке в соответствующей начальной схеме $S$.

Для построения алгоритмов решения задачи нумерации введем общие обозначения для указанных случаев в схемах $S$ и $S^{*}: N_{S}^{*}, N_{S^{*}}^{*}$ - номера их исходов и $R_{S}^{*}, R_{S^{*}}^{*}$ - их виды в этих схемах, которые обладают свойствами взаимной однозначности и парности.

Тогда пересчет результатов решения задачи нумерации схемы $S$ для схемы $S^{*}$ реализуется двумя следующими алгоритмами в прямой задаче нумерации (по данному $N_{S^{*}}^{*}$ найти $R_{S^{*}}^{*}$ ) и обратной задаче нумерации (по данному $R_{S^{*}}^{*}$ найти $N_{S^{*}}^{*}$.

АЛГОРИТМ РЕШЕНИЯ ПРЯМОЙ ЗАДАЧИ НУМЕРАЦИИ.

1) Приводим исходы схемы $S$ к виду исходов схемы $S^{*}$ (с повторами за счет уменьшения различий в них) и получаем исходы в том же количестве в порядке исходов схемы $S$;

2) в порядке исходов схемы $S$ в виде результата шага 1) считаем число $N^{*}$ сравнений каждого со всеми предыдущими в результате шага 1) до получения $N_{S^{*}}^{*}$-ного нового включительно;

3) по номеру $N^{*}$ из шага 2) по результату решения прямой задачи нумерации в схеме $S$ находим его вид $R^{*}$, совпадающий с искомым $R_{S^{*}}^{*}$ в схеме $S^{*}$.

АЛГОРИТМ РЕШЕНИЯ ОБРАТНОЙ ЗАДАЧИ НУМЕРАЦИИ.

1) Приводим исходы схемы $S$ к виду исходов схемы $S^{*}$ (с повторами за счет уменьшения различий в них) и получаем исходы в том же количестве в порядке исходов схемы $S$ (исходы $\{L\})$;

2) в порядке исходов схемы $S$ в виде результата шага 1) $\{L\}$ считаем число $N^{*}$ сравнений их видов с данным видом $R_{S^{*}}^{*}$ до первого с ним совпадения включительно;

3) маркируем часть исходов $\{L\}$ от первого до $N^{*}$-ного по их совпадениям и получаем исходы $\left\{L^{*}\right\}$;

4) находим искомый номер $N_{S^{*}}^{*}=\sum_{\left(L^{*}\right)} I\left(L^{*}\right)$, где $I\left(L^{*}\right)$ - индикаторная функция, определенная во введении, а суммирование проводится по множеству исходов $\left\{L^{*}\right\}$. 


\section{4. Вероятностные распределения на множествах исходов схем $\boldsymbol{A}, \boldsymbol{B}, \boldsymbol{C}, \boldsymbol{D}$}

Итерационный случайный процесс перечисления исходов комбинаторной схемы определяет все результаты ее анализа, в том числе и вероятностное распределение на множестве ее исходов. В рассматриваемых схемах размещения частиц по ячейкам он для каждой схемы состоит в последовательном поединичном добавлении ее элементов и задается структурой своего графа поитерационного перечисления ее исходов и вероятностями итерационных переходов из каждого его промежуточного состояния, определенным заданием которых управляется, т.е. приводит к разным вероятностным распределениям итоговых исходов каждой схемы. Здесь будем рассматривать случай «согласованности» вероятностных распределений всех схем группы, понимая под этим задание распределения вероятностей их итоговых исходов через вероятности итерационных переходов в процессе перечисления исходов начальной схемы как вероятностей их траекторий в графе. Установление вероятностных распределений исходов рассматриваемых схем группы состоит в их получении из распределения исходов начальной (базовой) схемы путем установления специфического соответствия между ее исходами и исходами каждой схемы по ее конкретным условиям.

В идее глобализации исследований таких комбинаторных схем, состоящей в возможности пересчета результатов анализа из схемы с большей дифференциацией исходов, являющейся базовой, будем рассматривать единую логику введения вероятностных параметров процессов схем данного класса. Она будет заключаться, например, в том, что каждая добавляемая в итерации частица равновероятно размещается по возможным в условиях схемы ячейкам. Множество исходов каждой схемы определяется по п. 2.3.1 из исходов близкой в группе схемы. А любые ограничения, общие для схем группы, учитываются удалением из графа базовой схемы лишних траекторий с пропорциональным пересчетом вероятностей итерационных переходов в ней в каждом пучке каждой его итерации с сохранением их суммарной единичной вероятности. Тогда на каждой итерации переходные вероятности формирования исходов процессами их перечисления в остальных схемах при отбраковках повторов будут иметь для каждого исхода суммарные вероятности их траекторий в графе перечисления до отбраковки.

Для примера рассмотрим процедуру пересчета исходов схем без ограничений $B, C, D$ и их вероятностей из схемы $A$ при $n=3, r=2$ с равновероятными итерационными переходами, что в ней означает равновероятное размещение каждой добавленной частицы по всем ячейкам схемы парами: из $A$ в $B$ (рис. 1); из $A$ в $C$ (рис. 2); из $A$ в $D$ (рис. 3 ). (Для схемы $D$ базовыми можно еще считать схемы $B$ и $C$.)

Сравним полученные исходы и их вероятности с непосредственным их нахождением по условиям схем $B, C, D$ перечислительным методом при равновероятном размещении каждой добавленной частицы в них по всем ячейкам (см. рис. 4-6).

Совпадение с ранее полученными последовательными пересчетами из начальных схем, начиная с $A$, результатов итоговых исходов схем $B, C, D$ и их вероятностных распределений на рисунках с их непосредственным исследованием перечислительным методом иллюстрирует логику расчета вероятностей 


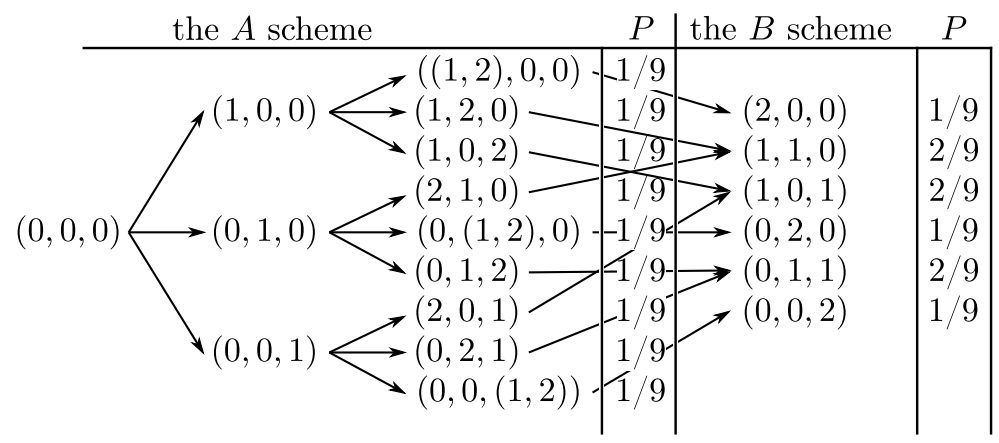

Рис. 1. Пересчет исходов схемы $B$ и их вероятностей из схемы $A$

[Figure 1. Recalculation of outcomes of the $B$ scheme and their probabilities from the $A$ scheme]

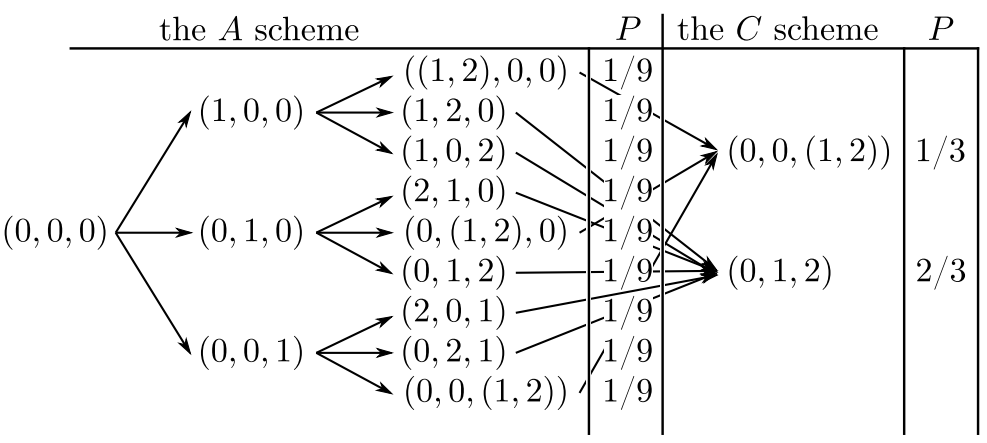

Рис. 2. Пересчет исходов схемы $C$ и их вероятностей из схемы $A$

[Figure 2. Recalculation of outcomes of the $C$ scheme and their probabilities from the $A$ scheme]

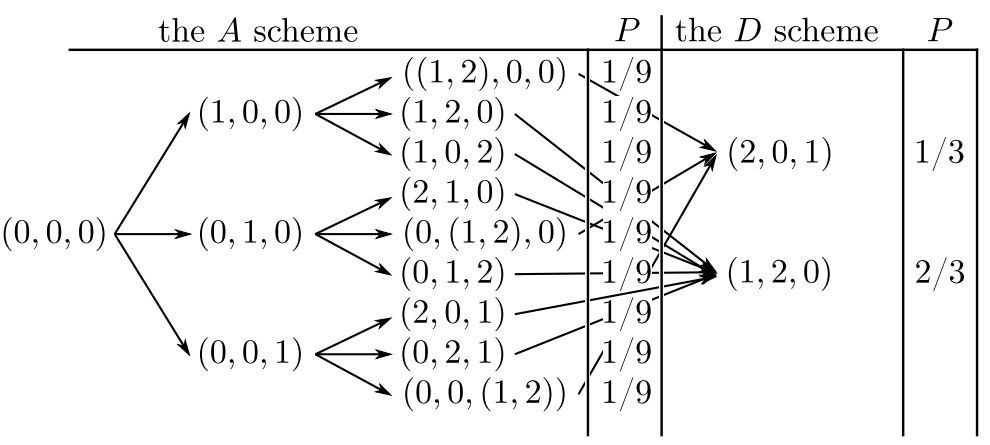

Рис. 3. Пересчет исходов схемы $D$ и их вероятностей из схемы $A$

[Figure 3. Recalculation of outcomes of the $D$ scheme and their probabilities from the $A$ scheme] 


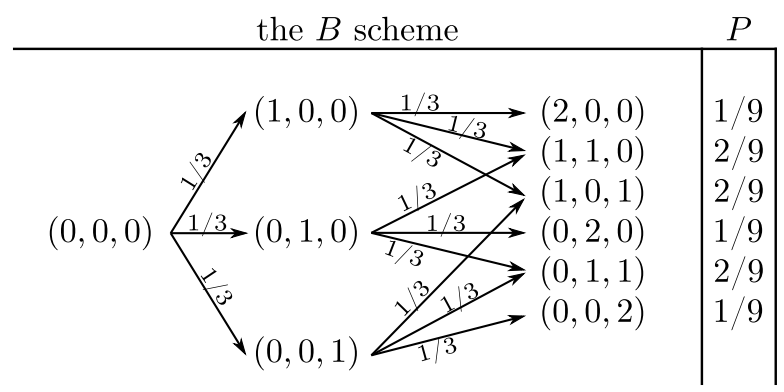

Рис. 4. Определение распределения и исходов схемы $B$ перечислительным методом

[Figure 4. Determination of the distribution and outcomes of the $B$ scheme by the enumeration method]

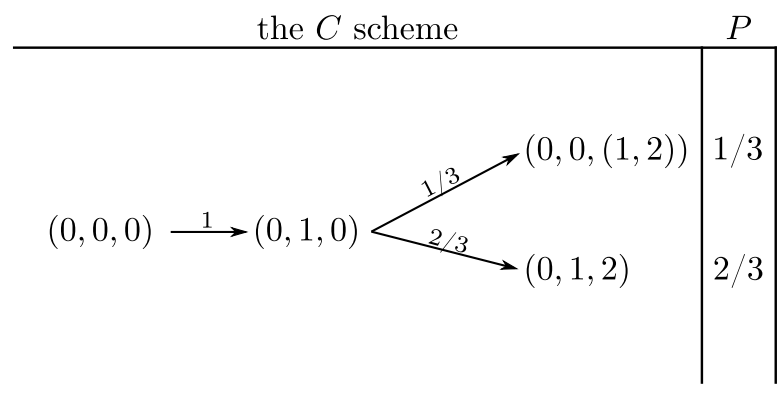

Рис. 5. Определение распределения и исходов схемы $C$ перечислительным методом

[Figure 5. Determination of the distribution and outcomes of the $C$ scheme by the enumeration method]

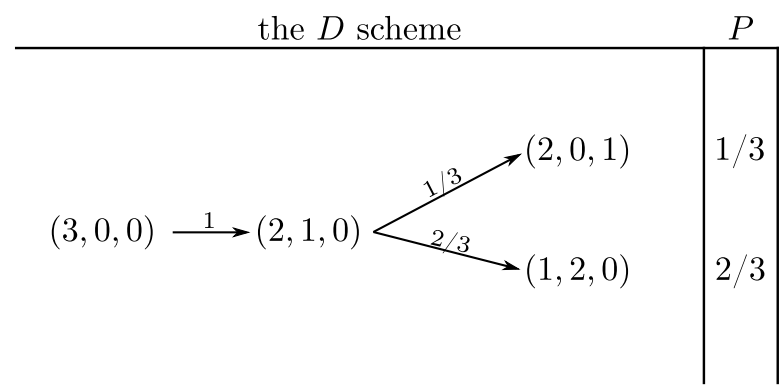

Рис. 6. Определение распределения и исходов схемы $D$ перечислительным методом

[Figure 6. Determination of the distribution and outcomes of the $D$ scheme by the enumeration method] 
итерационных переходов и итоговых вероятностных распределений исходов во всех схемах $A, B, C, D$ двумя способами.

\section{5. Моделирование исходов схем $B, C, D$}

По смоделированному исходу схемы $A$ (без или с ограничениями) для моделирования исхода каждой из схем $B, C, D$ при «согласованности» их вероятностных распределений достаточно применить к нему соответствующую процедуру приведения его к виду исхода изучаемой схемы по п. 1.

Кроме этого, моделирование каждого исхода изучаемой схемы $(B, C, D)$ по результату решения прямой задачи нумерации в каждой из них можно производить путем разыгрывания его номера одним случайным числом с полученным в ней из схемы $A$ при их «согласованности» распределением.

\section{Заключение (выводы)}

В глобализации анализа схем размещения частиц по ячейкам в группах с совпадающими ограничениями предложена организация пересчета результатов исследований из одной начальной схемы группы с наибольшей дифференциацией исходов для других схем группы последовательно уменьшением видов различимых в них элементов (ячеек и частиц) соответствующим набором алгоритмических преобразований без необходимости непосредственного учета специфики каждой схемы группы.

Характер пересчитываемых результатов определен перечислительным методом, формирующим исходы начальной схемы группы случайным процессом перечисления ее исходов с их управляемым вероятностным распределением. Это дает возможность более широкого их применения для схем размещения частиц по ячейкам в реальных процессах.

Конкурирующие интересы. Конкурирующих интересов не имею.

Авторская ответственность. Я несу полную ответственность за предоставление окончательной версии рукописи в печать. Окончательная версия рукописи мною одобрена.

Финансирование. Исследование выполнялось без финансирования.

\section{Библиографический список}

1. Виленкин Н. Я. Комбинаторика. М.: Наука, 1969. 328 с.

2. Riordan J. An Introduction to Combinatorial Analysis / Wiley Publicatlon in Mathematical Statistics. New York: John Wiley and Sons, 1958. x+244 pp.

3. Сачков В. Н. Введение в комбинаторные методы дискретной математики. М.: Наука, 1982. $384 \mathrm{c}$.

4. Сачков В. Н. Вероятностные методъ в комбинаторном анализе. М.: Наука, 1978. $320 \mathrm{c}$.

5. Hall M. Combinatorial Theory/ Wiley Classics Library. John Wiley and Sons, 1998. xviii +440 pp.

6. Ryser H. J. Combinatorial Mathematics / The Carus Mathematical Monographs. vol. 14. New York: John Wiley and Sons, 1963. xiv+154 pp.

7. Рыбников К. А. Введение в комбинаторный анализ. М.: МГУ, 1985. 312 с.

8. Колчин В. Ф. Севастьянов Б. А., Чистяков В. П. Случайные размещения/ Теория вероятностей и математическая статистика. Москва: Наука, 1976. 223 с.

9. Goulden I. P. Jackon D. M. Combinatorial Enumeration. Mineola, NY: Dover Publ., 2004. $\mathrm{xxvi}+569 \mathrm{pp}$. 
10. Энатская Н. Ю. Вероятностные модели комбинаторных схем // Вестн. ЮУрГУ. Сер. Матем. моделирование и программирование, 2020. T. 13, № 3. C. 103-111. https://doi . org/10.14529/mmp200312.

11. Энатская Н. Ю. Анализ комбинаторных схем в доасимптотической области изменения параметров // Труды Карельского научного иентра РАН, 2018. №7. С. 117-133. https://doi.org/10.17076/mat750.

12. Энатская Н. Ю. Комбинаторный анализ схемы размещения различимых частиц по различимым ячейкам без пустых ячеек// Труды Карельского научного иентра РАН, 2020. № 7. C. 120-126. https://doi.org/10.17076/mat1172. 


\title{
MSC: $60 \mathrm{~F} 15$
}

\section{Globalization of the analysis of particle placement models by cells}

\author{
(C) N. Yu. Enatskaya
}

National Research University "Higher School of Economics", Moscow Institute of Electronics and Mathematics,

34, Tallinskay st, Moscow, 123458, Russian Federation.

\begin{abstract}
The title of the paper means that its goal is a general approach to the pre-asmptotic analysis of schemes with different qualities in all combinations of their distinguishability of their constituent elements (cells and particles). To do this, in each group of such schemes with general restrictions, instead of directly studying them based on the specificity of each scheme, a certain general set of algorithmic procedures for recalculating the results of their pre-asymptotic analysis in the scheme is proposed, starting with the scheme with the greatest differentiation of their outcomes, sequentially for other schemes of the group with differences as one item. The analysis of each scheme is carried out according to the traditional and in a number of new following directions: constructing a random process of formation and numbered non-repeated enumeration of the outcomes of the scheme in the order of their receipt, finding their number, solving the numbering problem for the outcomes of the scheme, which consists in establishing a one-to-one correspondence between their types and numbers, setting their probabilistic distribution and modeling the outcomes of the scheme with this probabilistic distribution.

In particular, the cases of groups of schemes without restrictions on the placement of particles and with a restriction of at most one particle in a cell are studied separately, which lead to some well-known analytical results. Under any restrictions in the considered group of circuits, their analysis is carried out by implementing algorithmic procedures for sequential transformation of the results of the analysis of one circuit of the group for another. Combinations into such pairs of schemes are made on the basis of the difference in the quality of one of their elements.
\end{abstract}

Keywords: placement of particles into cells, pre-asymptotic analysis.

Received: 25 ${ }^{\text {th }}$ March, $2021 /$ Revised: $18^{\text {th }}$ August, $2021 /$

Accepted: $31^{\text {st }}$ August, $2021 /$ First online: $30^{\text {th }}$ September, 2021

\section{Research Article}

○ ()(i) The content is published under the terms of the Creative Commons Attribution 4.0 International License (http://creativecommons.org/licenses/by/4.0/)

Please cite this article in press as:

Enatskay a N. Yu. Globalization of the analysis of particle placement models by cells, Vestn. Samar. Gos. Tekhn. Univ., Ser. Fiz.-Mat. Nauki [J. Samara State Tech. Univ., Ser. Phys. Math. Sci.], 2021, vol. 25, no. 3, pp. 571-587. https://doi.org/10.14498/vsgtu1857 (In Russian).

\section{Authors' Details:}

Nataliya Yu. Enatskaya (10) https://orcid.org/0000-0003-1241-7543

Cand. Phys. \& Math. Sci.; Associate Professor; Dept. of Applied Mathematics;

e-mail: nat1943@mail.ru 
Competing interests. I have no competing interests.

Author's Responsibilities. I take full responsibility for submit the final manuscript to print. I approved the final version of the manuscript.

Funding. This research received no external funding.

\section{References}

1. Vilenkin N. Ya. Combinatorics. New York, Academic Press, 1971, xiii +296 pp.

2. Riordan J. An Introduction to Combinatorial Analysis, Wiley Publicatlon in Mathematical Statistics. New York, John Wiley and Sons, 1958, x+244 pp.

3. Sachkov V. N. Combinatorial Methods in Discrete Mathematics, Encyclopedia of Mathematics and its Applications, vol. 55. Cambridge, Cambridge Univ. Press, 1996, xiii +306 pp. https://doi.org/10.1017/cbo9780511666186.

4. Sachkov V. N. Probabilistic Methods in Combinatorial Analysis, Encyclopedia of Mathematics and its Applications, vol. 56. Cambridge, Cambridge Univ. Press, 1997, x+246 pp. https://doi.org/10.1017/CB09780511666193.

5. Hall M. Combinatorial Theory, Wiley Classics Library. John Wiley and Sons, 1998, xviii +440 pp.

6. Ryser H. J. Combinatorial Mathematics, The Carus Mathematical Monographs, vol. 14. New York, John Wiley and Sons, 1963, xiv+154 pp.

7. Rybnikov K. A. Vvedenie v kombinatornyi analiz [Introduction to Combinatorial Analysis]. Moscow, Moscow State Univ., 1985, 312 pp. (In Russian)

8. Kolchin V. F., Sevast'yanov B. A., Chistyakov V. P. Random Allocations, Scripta Series in Mathematics. New York, John Wiley and Sons, 1978, xi+262 pp.

9. Goulden I. P. Jackon D. M. Combinatorial Enumeration. Mineola, NY, Dover Publ., 2004, xxvi +569 pp.

10. Enatskaya N. Yu. Probabilistic models of combinatorial schemes, Bulletin of the South Ural State University, Ser. Mathematical Modelling, Programming and Computer Software, 2020, vol. 13, no. 3, pp. 103-111 (In Russian). https://doi.org/10.14529/mmp200312.

11. Enatskaya N. Yu. Analysis of combinatorial schemes in the pre-asymptotic region of parameter change, Proceedings of the Karelian Research Centre of the Russian Academy of Sciences, 2018, no. 7, pp. 117-133 (In Russian). https://doi.org/10.17076/mat750.

12. Enatskaya N. Yu. Combinatorial analysis of the scheme of allocation of distinguishable particles into distinguishable cells without empty cells, Proceedings of the Karelian Research Centre of the Russian Academy of Sciences, 2020, no. 7, pp. 120-126 (In Russian). https:// doi.org/10.17076/mat1172. 